\title{
Fuzzy Logic Model for Analysis of Computer Network Quality of Experience
}

\author{
Walter B. Kihuya \\ Institute of Computer \\ Science and Information \\ Technology, \\ Jomo Kenyatta University \\ of Agriculture and \\ Technology, \\ Mombasa, Kenya.
}

\author{
Dr. Calvins Otieno \\ Institute of Computer \\ Science and Information \\ Technology, \\ Jomo Kenyatta University \\ of Agriculture and \\ Technology, \\ Nairobi, Kenya.
}

\author{
Dr.Richard Rimiru \\ Institute of Computer \\ Science and Information \\ Technology, \\ Jomo Kenyatta University \\ of Agriculture and \\ Technology, \\ Nairobi, Kenya.
}

\begin{abstract}
The estimation of the QoE provides valuable input in order to measure the user satisfaction of a particular service/application. Network QoE estimation is challenging as it tries to measure a subjective metric where the user experience depends on a number of factors that cannot easily be measured. All the Network analysis models can be divided into two major groups: qualitative and quantitative. In recent years many quantitative models have been developed in terms of quantitative measures i.e. use of scale of numbers between 1 to 5 to represent user perception of QoS. The challenge with this model is where user perception is subjective and not precise thus cannot be clearly measured using quantitative methods. On the other side qualitative models are in early stages of exploration. Little has been done on qualitative methods. Basing on previous studies, few models exists that measure qualitative analysis of computer network quality of experience. However none incorporated all the four parameters of integrity of service; throughput, delay, packet loss and jitter as parameters of network QoE. The study's objective is to address this gap by proposing a fuzzy logic model for analysis of computer network QoE. The tools used in the study are Linux MTR tool for data extraction, Ms. Excel for data cleaning and presentation, Visual paradigm for constructing of Unified Modeling language diagrams, mat lab software for plotting of functions/data, implementation of algorithms and creation of user interfaces. Experimental research design and sampling mechanisms is applied for 15 samples. The methodology in use is fuzzy logic. In order to deal with fuzziness associated with linguistic variables, inference rules are introduced. Five input linguistic terms are identified: Very High, High, Medium, Low and Very Low. Five output linguistic terms are defined to describe the opinion scores: Excellent, Good, Fair, Poor and Bad. Four variables are used: delay, jitter, packet loss and throughput. This results to a total of 625 rules $\left(5^{\wedge} 4\right)$. The rules are further condensed to 240 logical rules basing on expert knowledge. The collected data was used for simulation in matlab environment basing on the 240 rules. The results shows, analysis of Computer network QoE is subjective in nature rather than objective thus requires a resilient mechanism like fuzzy logic in order to capture clear-cut results to be used for decision making. The target population for this model is the ISPs' clients. This will enable ISPs to have the best responsive measures to deal with clients' QOE parameters so as to meet the QOS as per SLAs.
\end{abstract}

Keywords: fuzzy logic, ISPs (Internet Service Providers), quality of experience (QoE), Quality of service (QoS), SLAs (Service Level Agreement)

\section{INTRODUCTION.}

QoE in the context of telecommunications networks is defined as the degree of delight or annoyance of the user of an application or service. It results from the fulfillment of his or her expectations with respect to the utility and / or enjoyment of the application or service in the light of the user's personality and current state. [1]

QoE is defined by International Telecommunication Union: $I T U$ as the overall acceptability of an application or service, as perceived subjectively by the end-user.

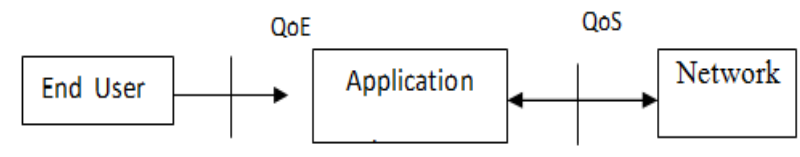

Figure2. 1: Relationship between QoS and QoE

Fuzzy logic is a natural, continuous logic patterned after the approximate reasoning of human beings [3]. As a theory mathematical discipline, fuzzy logic reacts to constantly changing variables [3].It challenges traditional logic by not being restricted to the conventional binary computer values of zero and one. Instead, fuzzy logic allows for partial truths and multivalued truths [3] .Fuzzy logic is especially advantageous 
for problems that cannot be easily represented by mathematical modeling because data is either unavailable or incomplete or the process is too complex [3].The real world language used in fuzzy control enables engineers to incorporate ambiguous, approximate human logic into computers using linguistic modeling, as opposed to mathematical modeling, greatly simplifies the design and modification of a fuzzy logic system [3].

Fuzzy set theory [4]was developed to address contexts in which decision Makers need to accurately analyze and process information that is imprecise in nature.

Fuzzy sets provide a conceptual framework, as well as an analytical tool to solve real World problems where there is a lack of specific facts and precision [4].

Human semantics are embedded in the meaning of fuzziness and comparison on the other hand; the usage of multi granularity linguistic information can eliminate the difference from evaluators [5].

Using fuzzy logic algorithms could enable machines to understand and respond to vague human concepts such as hot, cold, large, small, etc. It also could provide a relatively simple approach to reach definite conclusions from imprecise information [3].

The information technology (IT) and electronics industries apply the QoE model to businesses and services because QoE depends on customer experience; assessments are compiled from large user group polls. The most commonly used model is mean opinion score (MOS). The MOS is expressed as a single rational number, typically in the range $1-5$, where 1 is lowest perceived quality and 5 is the highest perceived quality. Other MOS ranges are also possible, depending on the rating scale that has been used in the underlying test. This model is thus quantitative in nature while user perception is subjective and not precise thus cannot be clearly measured using quantitative methods. [2]

Fuzzy set theory was first introduced by Zadeh in 1965 . Fuzzy logic is a problem solving methodology that provides a simple way of definite conclusions from vague and imprecise information. He was motivated by observing that human reasoning can utilize concepts and knowledge that don't has well [5].
In the case of Network analysis, all Network analysis models can be divided into two major groups: qualitative and quantitative. Qualitative metrics do not own quantitative values and cannot be measured numerically. Purposely, linguistic terms are used to evaluate performance of qualitative metrics [6]. Fuzzy logic controller is useful when the problem is too difficult to be solved with quantitative approaches [4].

\section{RELATED WORK.}

Several researches have been done on fuzzy logic in relation to quality performance though little has been done on fuzzy logic model for analysis of quality of experience.

The study in [7] proposed a Fuzzy logic aggregation of wireless sensor network data for smart traffic light control. This approach uses smart traffic control systems (STCS) to make traffic routing decisions. STCS use real time data and mimic human reasoning thus prove promising in vehicle traffic control. This presents a smart traffic light controller using fuzzy logic and wireless sensor network (WSN). The approach is designed for an isolated four way roundabout. It employed fuzzy logic to control the lights and determine how the green light will be assigned for each approach. The WSN collected the traffic data in real time. This data is aggregated and fed into a fuzzy logic controller (FLC) in form of two inputs - traffic quantity (TQ) and waiting time (WT) for each approach. Based on the inputs, the FLC then computes an output priority degree (PD) that controls green light assignment. Using the $\mathrm{PD}$, an algorithm is formulated that assigns green light to the lane with highest PD. The cycle continues until all approaches get green.

In [8] a research study on a Fuzzy Logic System for Evaluating Quality of Experience of Haptic-based Applications was proposed. The proposed taxonomy was modeled with a fuzzy logic system and finally was tested by a Mamdani fuzzy inference system. In the mentioned study, by making some assumption like rule selection and membership function selection, the effect of different perception measures parameters such as rendering quality, physiological and psychological was studied. Here, fuzzy logic system was applied for objective measuring of QoE parameters.

The research work in [9] exhibited QoE estimation for web service selection using a Fuzzy-Rough hybrid expert system. 
A methodology to estimate the quality of web services based on a fuzzy-rough hybrid algorithm is proposed. The estimated web QoE is used to select the most performing service among different web services. Fuzzy expert systems are good at making decision with imprecise information; however, they cannot automatically formulate rules that they require for making the decisions. Therefore, a fuzzy-rough hybrid expert system is proposed in this study where rough set theory is used to define the rules necessary for the fuzzy expert system. Three QoS parameters: reliability, execution time (in seconds), and availability (in seconds) are measured during the performance of the tests. Input linguistic terms are: Low, Medium and High. The output linguistic terms in use are: Bad, Poor, Fair, Good and Excellent.

The research work in [10] proposed analysis of Quality of Experience by applying Fuzzy logic: A study on response time. In this work, with a fuzzy perspective, the effect of response time variation in a network on the quality perceived by users is shown. Later, shows how by applying fuzzy techniques the linguistic terms and the users' perception can be translated into quantitative values. The main objective of this project was to analyze the fuzziness of QoE in order to provide more understandable user perception. This included proposing response time performance criteria that correlate well with QoE measurement result presented by fuzzy concepts. The proposed methodology provides a fuzzy relationship between QoE and Quality of Service (QoS) parameters. To identify this fuzzy relationship a new term called Fuzzi ed Opinion Score (FOS) representing a fuzzy quality scale is introduced. A fuzzy data mining method is applied to construct the required number of fuzzy sets. Then, the appropriate membership functions describing fuzzy sets are modeled and compared with each other. The proposed methodology intended to assist service providers for better decision-making and resource management [10] .

In [11] an efficient algorithm for transmitting packet for better quality of service in adhoc mobile network was proposed. In this study, Fuzzy Self Organizing Map (FSOM) provide very efficient algorithmic tools for transmitting packet in an efficient manner by taking the most efficient route and also the bandwidth, latency and range network parameters are considered to determine how good is the data delivered. The results indicated that fuzzy logic can guarantee QoS of every packet in the network. Incorporation of fuzziness in the input and output of the proposed model was seen to result in better performance. Input variables were only three properties: low, normal, and high. The output variables were poor, good and excellent.

In [12], a fuzzy logic based approach is in use for maintaining VoIP Quality in a network which is affected by many network factors (packet loss, packet delay, and jitter).In this case, Resource Reservation Protocol application was configured to control Token Bucket Algorithm and the simulation experiments were carried out with Opnet. In addition, comparison between Token Bucket with and without Quality of Service aimed at measuring network factors was performed. In this paper, building Fuzzy Token Bucket System consists of three variables (Bandwidth Rate, Buffer Size, and New Token) in order to improve Token Bucket Shaper output variable (New Token) by Fuzzy Stability model for Voice over IP quality maintaining. The linguistic values in use for each variable were: Buffer Size \{VL, L, M, H, and VH\}, Bandwidth Rate \{VL, L, AL, BA, AV, AA, BH, H, and $\mathrm{VH}\}$ and New Token $\{\mathrm{VL}, \mathrm{L}, \mathrm{BA}, \mathrm{AV}, \mathrm{AA}, \mathrm{H}$, and $\mathrm{VH}\}$

The study in [9] revealed the analysis of the impact of different network QoS parameters on users perceived video QoE for VoD (Video-on-Demand) services. Network parameters in use included: Packet loss rate, Burst packet loss and Jitter. The input linguistic terms involved were Very annoying, slightly annoying, Imperceptible, Annoying and perceptible but not annoying. The output linguistic terms in use were Very annoying, slightly annoying, Imperceptible, Annoying and perceptible but not annoying. This study proposed a methodology based on a fuzzy expert system to objectively estimate the video QoE. To validate the methodology, the developed system was integrated as part of a monitoring tool in an industrial IPTV (Internet Protocol Television) test bed and compared its output with standard Video Quality Monitoring (VQM). The evaluation results show that the proposed video quality estimation method based on fuzzy expert system can effectively measure the network impact on the QoE.

\section{MOTIVATION.}

In recent years many network analysis models have been developed in terms of quantitative measures. This mechanism 
is quantitative in nature (use of scale of numbers between 1to5) to represent user perception of QoS. The challenge with this model is where user perception is subjective and not precise thus cannot be clearly measured using quantitative methods. The qualitative model is in early stages of exploration thus little have been done on this research.

Few models exists that measure qualitative network QoE but none incorporated all the four integrity of service parameters. Therefore, this study is inspired to address this gap by presenting an alternative approach of measuring network QoE parameters under integrity of service which measures underlying network QoS related parameters (throughput, packet loss, delay and jitter) by using fuzzy logic concept.

\section{OBJECTIVES.}

\subsection{General Objectives}

To design a Fuzzy logic model for Analysis of computer Networks Quality of Experience.

\subsection{Specific Objectives}

i) To analyze the fuzziness of Network QoE in order to provide more understandable user perception.

ii) To design a fuzzy logic framework for analysis of computer networks Quality of Experience through developing linguistic terms and variables, designing fuzzy membership function for different linguistic terms, designing fuzzy rules ,fuzzification and defuzzification

iii) To develop a fuzzy logic framework for analysis of computer networks Quality of Experience.

iv) To test the performance of network QoE and estimate the variation of user satisfactions level in function of network integrity of service parameters.

v) To implement the developed framework for use by end users. model for analysis of computer networks Quality of Experience?

3) What are the methodologies to device a method to estimate the variation of the user satisfaction level in function of the network QoS conditions?

4) Which relevant mechanisms are in place to test the performance of network QoE and estimate the variation of user satisfactions level in function of network integrity of service parameters?

5) Which techniques are used to implement the developed framework for use by end users?

\section{RESEARCH METHODOLOGY.}

This phase covers Research Design, Data Collection procedure involved in the study, Data analysis based on Research Methodology.

\subsection{Research Design:}

This study focuses on the fuzzy logic model for analysis of computer network quality of experience, a study on integrity of service.

The research approach is experimental Research Design. This allows the researcher to have complete control over the extraneous variables \& can predict confidently that the observed effect on the dependable variable is only due to the manipulation of the independent variable.

Experimental research is often used where there is time priority in a causal relationship (cause precedes effect), there is consistency in a causal relationship (a cause will always lead to the same effect), and the magnitude of the correlation is great.

The causal relationship in this case is between four variables. The variables are underlying network Qos-related parameters (Throughput, delay, packet loss and Jitter) which lay under integrity of service Network QoE parameters whose manipulation will affect the output.

\subsection{Data Collection:}

In a nutshell, activities involving data can be grouped into Data extraction, Data processing/ cleaning, data presentation and data analysis based on research methodology. 


\subsubsection{Data Extraction:}

Data collection procedure involves an experiment for data extraction from a network setup. Data is extracted from 15 Autonomous Systems (AS)/ network connections for 19 services at an interval of 5 minutes using Linux mtr tool. This data is acquired inform of TXT file (.txt) which can be viewed by notepad ++ tool.

There is a vast challenge when it comes to data extraction of network QoE data. These factors ranges from the type of tool to use, the kind of data to acquire, the method to use for data cleansing to make it relevant for use etc.

The command to use in Linux mtr tool to capture the required data for dataset is mtr -rw -o "DRAM". For instance,For the case of mtr command to access Gmail's Dropped packets, Received packets, Average RTT/Delay and Jitter Mean/Avg is accessed by below order of fields:

\$ mtr -rw -o "DRAM" --aslookup www.gmail.com Whereby the initials represent:

mtr-My Traceroute.

rw- Report wide mode. Without the --report option, mtr will run continuously in an interactive environment. The interactive mode reflects current round trip times to each host. In most cases, the --report mode provides sufficient data in a useful format.

O-In the order of e.g. In the order of DRAM.

DRAM- Dropped packets, Received packets, Average RTT/Delay and Jitter Mean/Avg respectively.

aslookup will be used to display Autonomous systems.

This will produce the following output:

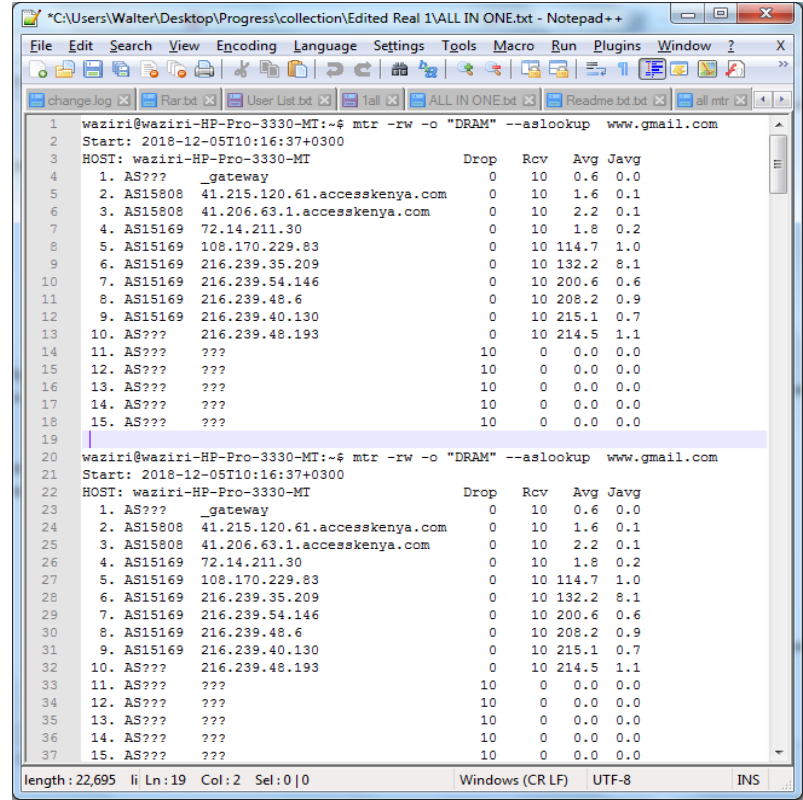

Figure 4.12: Mtr output in Ubuntu Linux platform.

Each numbered line in the report represents a hop. Hops are the Internet nodes that packets pass through to get to their destination. They are also referred to as Network Connections/Autonomous systems in the network initialized by "AS".

In the case where we have "AS???" in the Autonomous systems, it's an indication that:

The question marks appear when there is no additional route information.

Sometimes as a result of a poorly configured router will send packets in a loop.

\subsubsection{Data Processing/Data Cleaning:}

The acquired data from Linux Mtr tool is exported to Ms. Excel for cleaning/ processing. The processed data is obtainable in form of excel workbook format (.xlsx) each having crisp values for Delay, jitter, packet loss, throughput and users dataset.

\subsubsection{Data Presentation:}

Each column field from mtr txt data file is given a different excel workbook for different dataset.

This results into four workbook in excel each having packet loss, Throughput, Delay, and jitter datasets as shown below. The fifth extra workbook will be used to display the User List i.e. Network Connections/Autonomous systems in the network. 
The obtained crisp values for Delay, jitter, packet loss and throughput are used as input dataset for developed framework.

The values are organized into tables each arranged in a matrix of $15 * 19$ as shown below to indicate the experiment was carried out on 15 autonomous systems/network connections, 19 times at an interval of 5 minutes each.

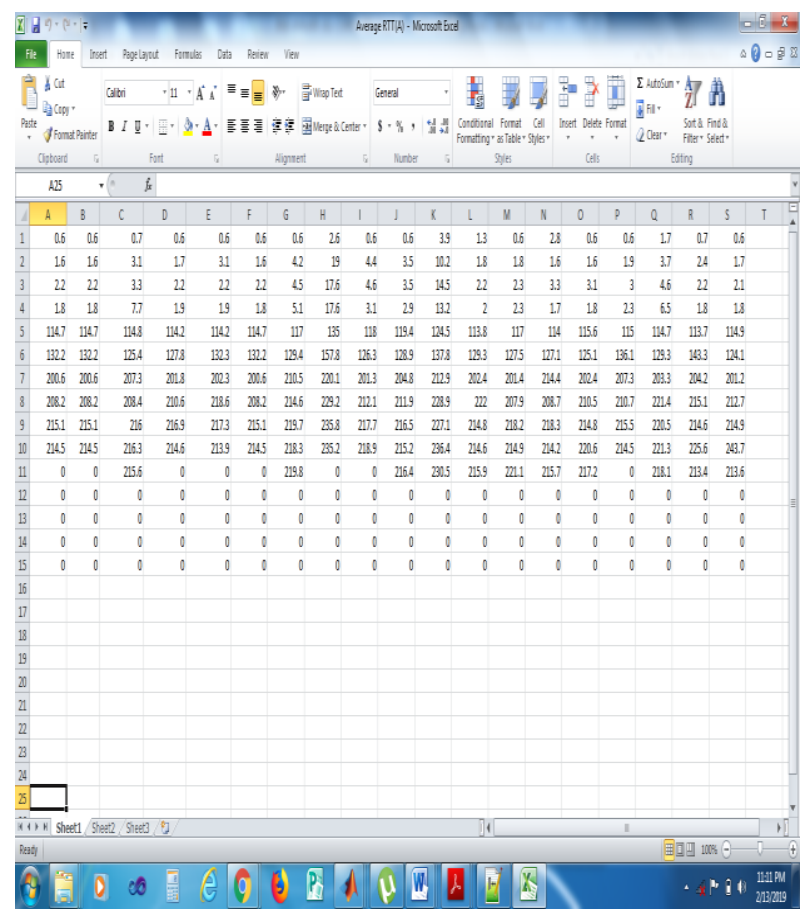

Figure 3.3: Processed data for Delay (A)

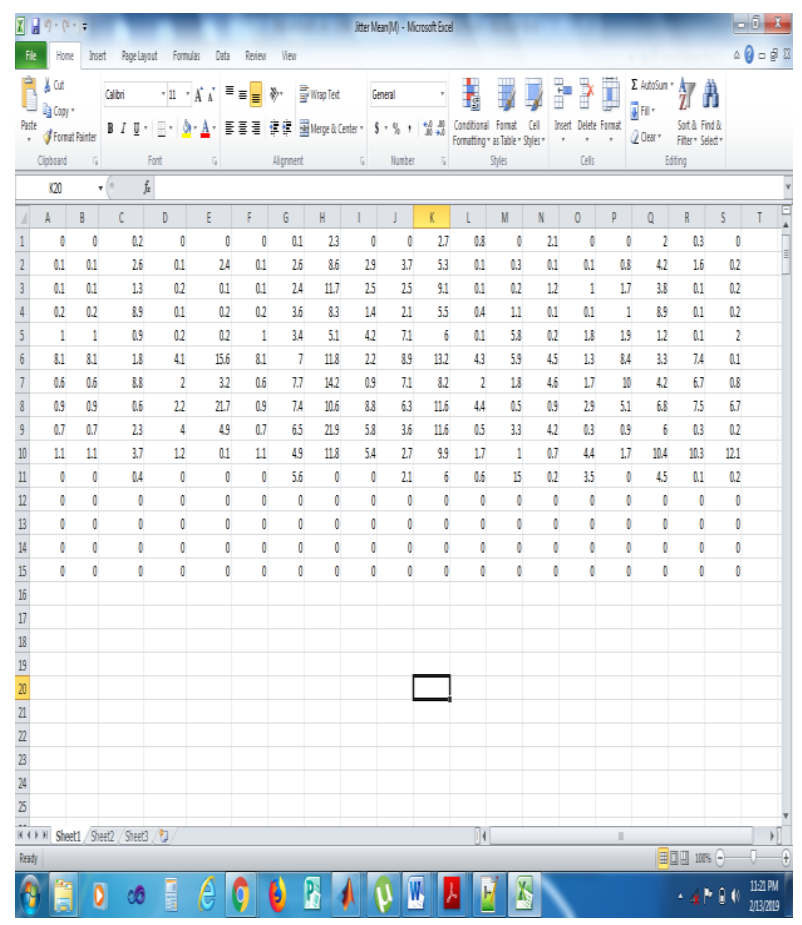

Figure 3.4: Processed data for Jitter Mean (M)

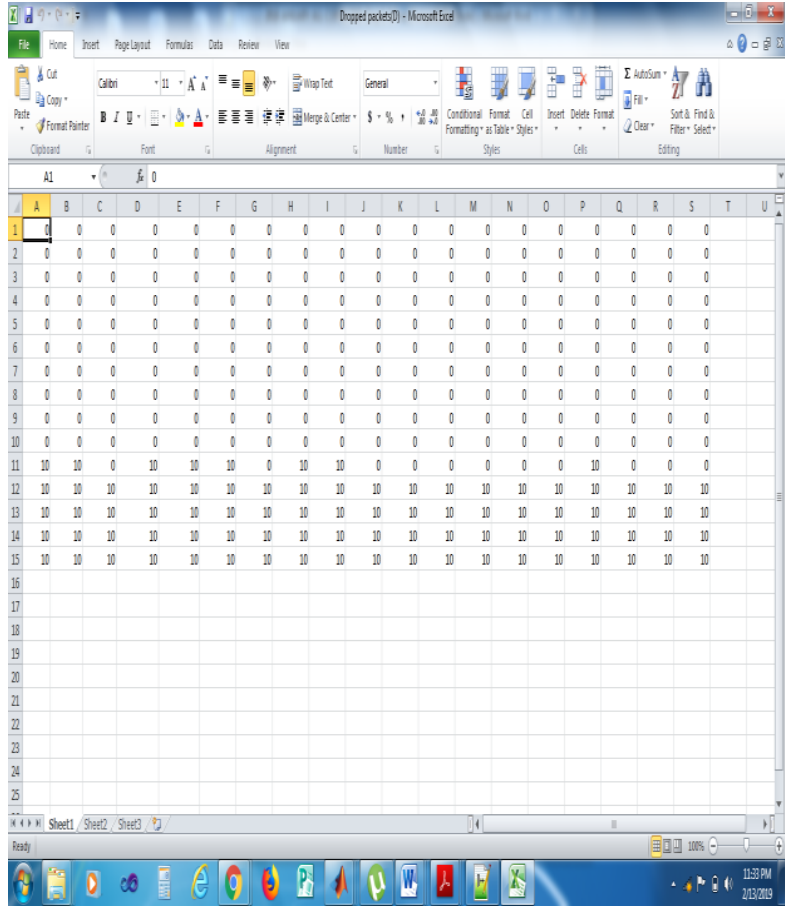

Figure 3.5: Processed data for Packet Loss/Dropped packets (D)

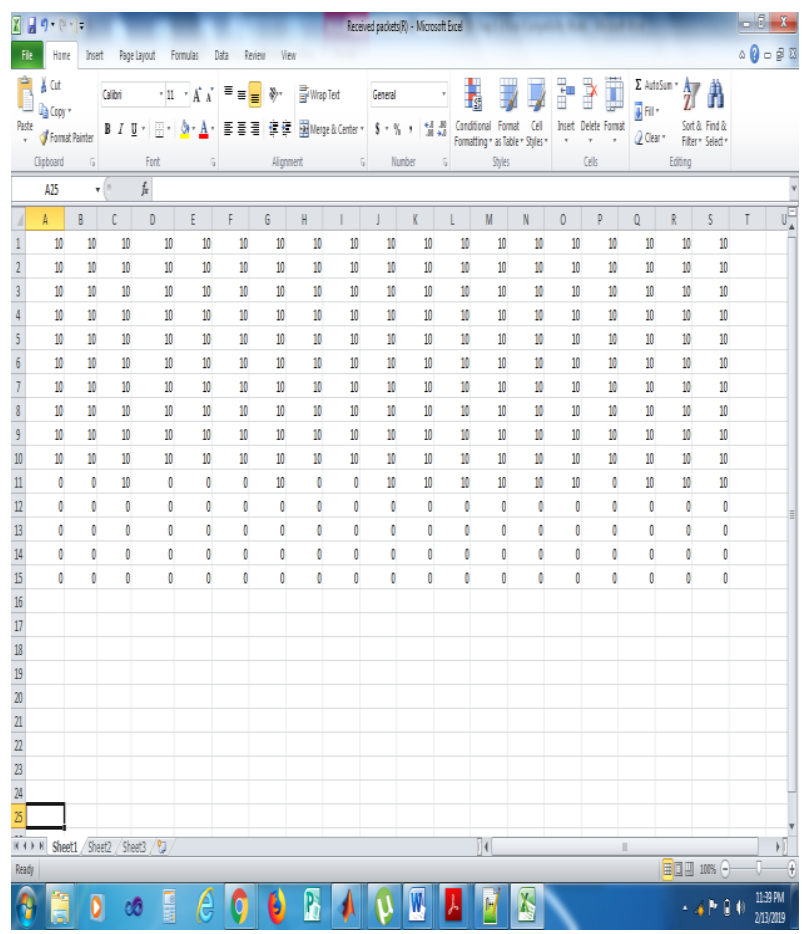

Figure 3.6: Processed data for Received Packets $(\mathbf{R})$ 


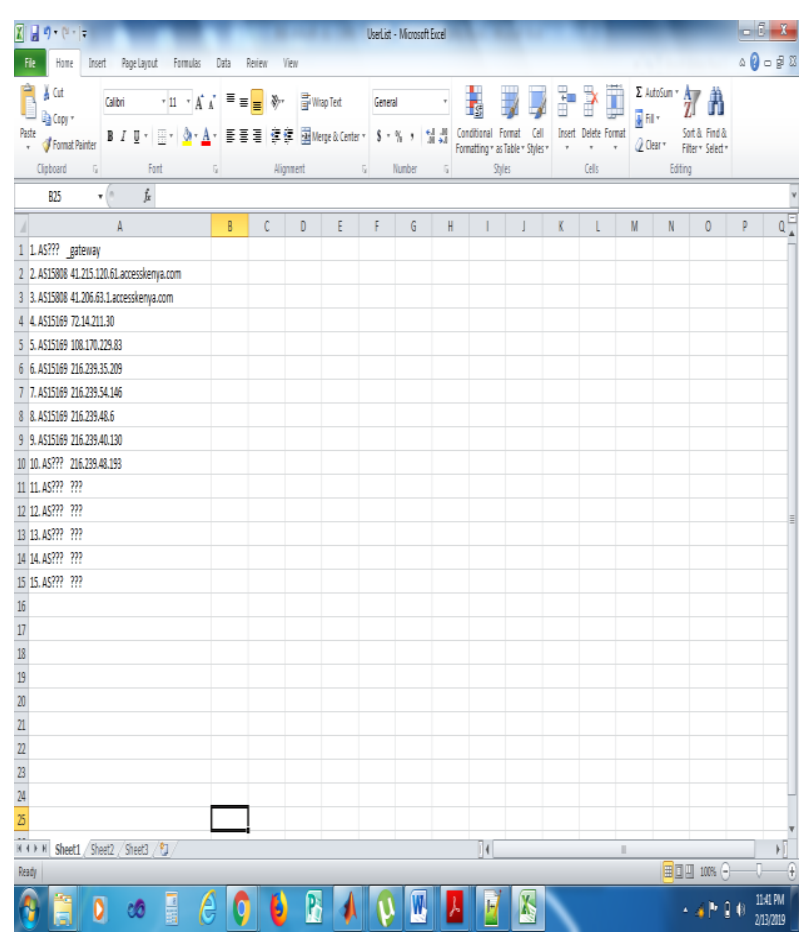

Figure 3.7: User List (Autonomous systems/Network Connections)

\subsection{Data Analysis Based on Research Methodology:}

The acquired data from designed experiment is used to construct the proposed framework of fuzzy logic model for analysis of computer network quality of experience. The data is used as input variables data of the framework to make rational analysis based on the membership function and fuzzy rules.

Data analysis is guided by fuzzy logic Methodology framework design process/guidelines. The methodology is grouped into four phases: Initialization, Fuzzification, Inferencing and defuzzification.

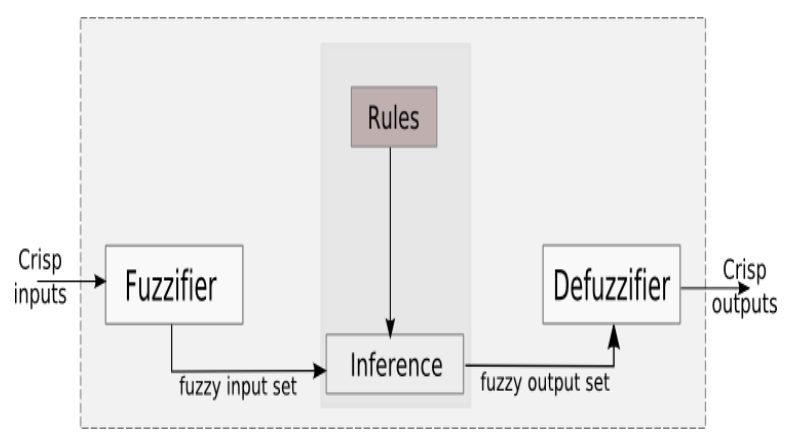

Figure 3.3: Fuzzy Logic System [13].

\subsubsection{Initialization:}

This phase comprises of defining the linguistic variables and terms, constructing the membership functions and constructing the rule base.

Five linguistic terms are identified for use i.e. "Excellent", "Good", "Fair", "Poor", and "bad". Four variables for network integrity of service QoE parameters are identified i.e. Throughput, Delay, Delay variation/Jitter and packet loss. These are the primary factor for QoS quantification of any network according to [14].

Constructing the membership functions (MF) is done at this stage by determining a curve that outlines how each point in the input universe is plotted to a membership value (or degree of membership) between 0 and 1.A triangular membership function is used to acquire the notch of membership for each linguistic term because of its computational efficiency.

Moreover, the initialization phase involves constructing the rule base. The identified Five linguistic terms and the Four variables for network integrity of service QoE parameters results into 625 rules $\left(5^{\wedge} 4\right)$. The rules are further dropped to 240 rules basing on expert knowledge by discarding the illogical rules thus remaining with logical rules to make rational decisions.

The illogical is as a result whereby some conditions cannot exist at the same time for instance in rule 1 of the 625 rules indicates:

1. If delay is very low, jitter is very low, packet loss is very low and throughput is very low then User Satisfaction N/A.

This rule is N/A thus illogical since when delay, jitter and packet loss are very low then throughput is supposed to be high or very high in ideal network situation as these three variables which are supposed to make the throughput very low, their existence too are very low not to certain levels to affect the network throughput to match being very low.

\subsubsection{Fuzzification:}

A crisp set (subset elements of the set, definitely do belong to the set), of input unprocessed information is assembled and transformed to a fuzzy set (sets whose elements have degrees of membership) by using fuzzy linguistic variables, membership functions and fuzzy 
linguistic terms through fuzzification [3]. This is achieved by Fuzzifier component of the fuzzy Logic System.

\subsubsection{Inference:}

This stage involves evaluating the rules in the rule base. Each rule follows the order to fulfill certain condition. The logical 240 rules are interpreted one after the other. This is achieved by Fuzzy Inference system component of the Fuzzy Logic System. In this work, Mamdani fuzzy inference system is used to achieve the inferencing in the developed framework.

The Fuzzy set operator "AND" is used to aggregate the output of each rule. The results of each rule are combined at this phase. The matched fuzzy rules are then used in the defuzzification process.

The properties of logical "AND" and "OR" makes the result of a logical expression to be sometimes fully determined before evaluating all of the conditions.

The logical operator "AND" is selected for connecting the inputs in this experiment since the operator returns logical 0 (false) if even a single condition in the expression is false in an ideal situation. For instance in one of the 625 rules:

\section{Example 1:}

If delay is very low, jitter is very low, packet loss is very low and throughput is very high then User Satisfaction EXCELLENT:

In an ideal situation, when delay, jitter and packet loss are very low then throughput is very high as the network suffers no hitches thus resulting to user satisfaction being excellent.

\subsubsection{Defuzzification Of the Output:}

The linguistic variables \& terms are matched, fuzzy rules generated and the output results obtained for each parameter are aggregated into one crispvalue through defuzzification.

Five linguistic terms considered at this phase include "Excellent", "Good", "Fair", "Poor", and bad. The linguistic terms are quantified on a numerical scale on a range of $5,4,3,2$ and 1 respectively whereby the higher the value, the better the $\mathrm{QoE}$ and the lower the value the worse the QoE.

This process involves producing a quantifiable result in Crisp logic, given fuzzy sets and corresponding membership degrees.

Moreover this process maps a fuzzy set to a crisp set. It is typically needed in fuzzy control systems. These will have a number of rules that transform a number of variables into a fuzzy result, that is, the result is described in terms of membership in fuzzy sets [13]. This is the purpose of the defuzzifier component of a FLS.

In this work, weighted average method is the defuzzification technique to use because of its computational efficiency.

Graphical tools to build, edit and view fuzzy inference systems:

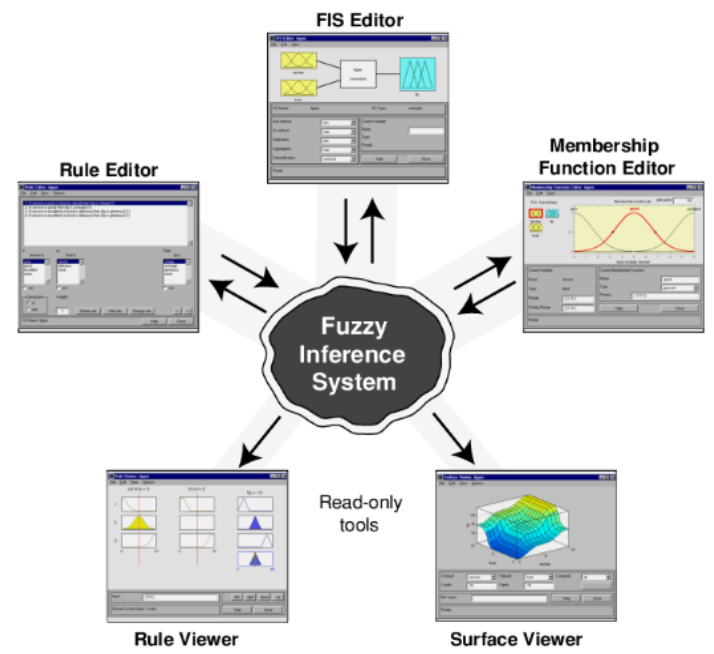

Figure 4.2: Graphical tools for fuzzy inference systems.

\section{ANALYSIS/ DISCUSSIONS OF THE RESULTS.}

Unit of analysis is based on the objectives achieved. This research achieved the following specific objectives:

\subsection{To Analyze the Fuzziness of Network QoE In Order To Provide More Understandable User Perception:}

This objective was achieved by demonstrating the fact that user satisfaction is not precise rather its subjective in nature thus use of linguistic terms to categorize the fuzzy sets in terms of the level of user satisfaction : Very Low, Low, High, Medium, and Very High. The use of membership function to determine the degree of relationship between the linguistic terms and the linguistic variables demonstrates fuzziness i.e. all information in fuzzy set whether the elements in fuzzy sets are discrete or continuous.

Moreover there is no specific value to determine User satisfaction level thus the use of Triangular membership 
function was effective to manipulate the range of values for each record set for instance "GOOD" Network QoE Membership function has a range of values from [2.5 3.75 5] whereby 2.5 is the lowest value that someone can rate the QoE as Good, 3.75 is the mean value while 5 is the highest value for this membership.

Likewise, the use of "IF THEN" statement in fuzzy rules to join the different imprecise input parameters in order to obtaining a single output demonstrates the fuzziness in network QoE. The rules for defining fuzziness are fuzzy too.

Furthermore, the need to use either AND, OR and NOT operators of Boolean logic that exists in fuzzy logic for the purpose of manipulation of different input values in order to obtain an output value demonstrates the fuzziness of network QoE. For instance in this work, AND operator is used to aggregate the fuzzy rule outputs.

\subsection{To Design a Fuzzy Logic} Framework For Analysis Of Computer Networks Quality of Experience Through Developing Linguistic Terms \& Variables, Designing Fuzzy Membership Function For Different

\section{Linguistic Terms, Designing Fuzzy}

\section{Rules:}

This activity achieved the following results: identification of five input linguistic terms: Very Low, Medium, Low, High and very High), Four input variables (Delay, Jitter, Throughput and packet Loss), five output linguistic term (Excellent, Good, Fair, Poor and Bad), designing Triangular membership function for different linguistic terms, designing 625 fuzzy rules $\left(5^{\wedge} 4\right)$ which were later reduced to 240 logical rules to be fired in the experiment.

Fuzzy rules operate using a sequence of if-then statements. For instance in this work: if delay is very low, jitter is very low, packet loss is very low and throughput is high then User Satisfaction GOOD.

The AND, OR, and NOT operators of Boolean logic exist in fuzzy logic, usually defined as the minimum, maximum, and complement; when they are defined this way, they are called the Zadeh operators, because they were first defined as such in Zadeh's original papers [4] In this work, AND operator is used to aggregate the fuzzy set values in order to acquire the output.

From the membership functions, we can calculate the truth value of each fuzzy proposition and of the fuzzy conjunction the minimum degree of membership is taken as the output when AND operator is used. In a nutshell, the design stage happened to design the following components:

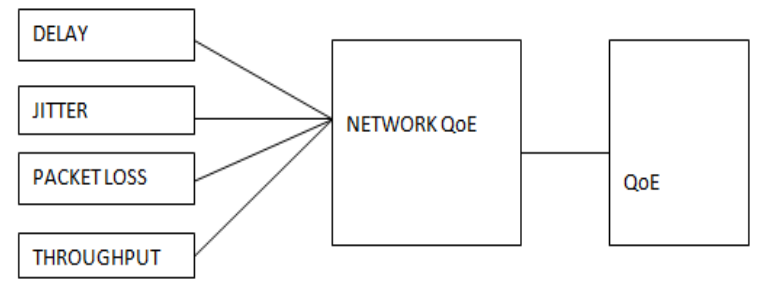

Figure 4.10: Designed Network QoE framework.

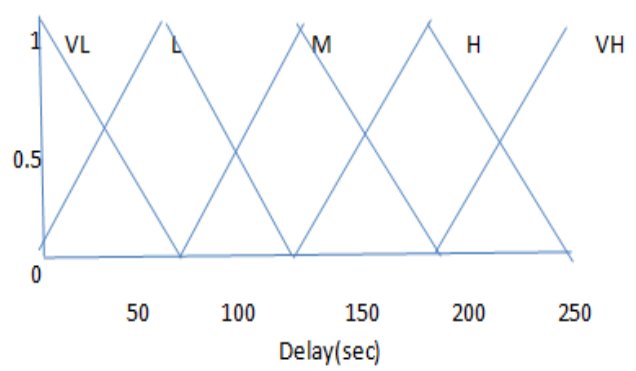

Figure 4.11: Designed Triangular membership

function for Delay input linguistic term.

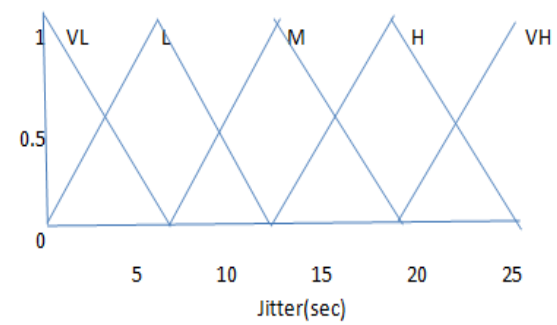

Figure 4.12: Designed Triangular membership function for Jitter input linguistic term.

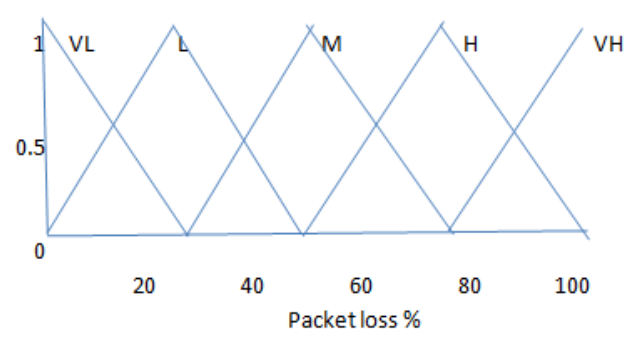

Figure 4.13: Designed Triangular membership function for Packet loss input linguistic term. 


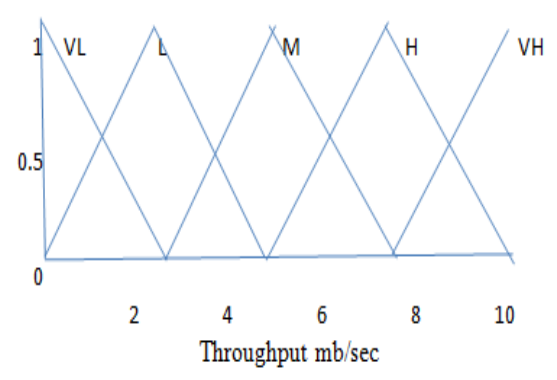

Figure 4.14: Designed Triangular membership function for Throughput input linguistic term.

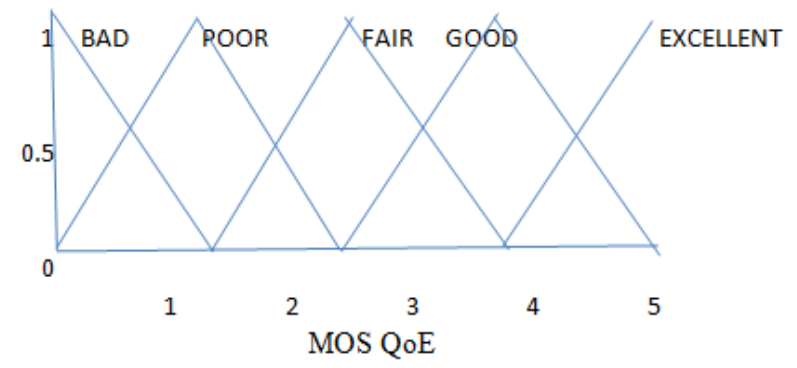

Figure 4.15: Designed Triangular membership function for different output linguistic terms.

7.3 To Develop a Fuzzy Logic Framework For Analysis Of Computer Networks Quality Of Experience:

The following was achieved in this activity: The designed framework was developed by use of fuzzy logic methodology. At this juncture, whatever was designed in the design phase is being developed in matlab environment.

In this scenario, the collected crisp values data are converted into fuzzy sets through fuzzification. The output fuzzy sets are further converted into a single crisp value through weighted average defuzzication technique. The acquired value is used for analysis of computer networks Quality of Experience.

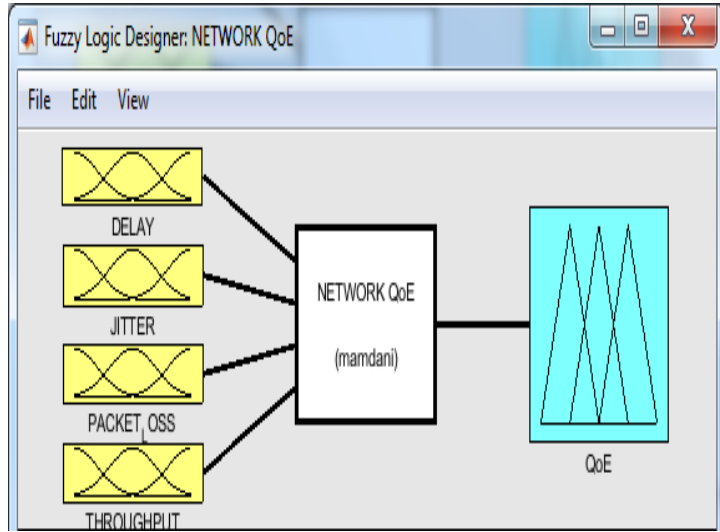

Figure 4.16: Developed Network QoE framework.

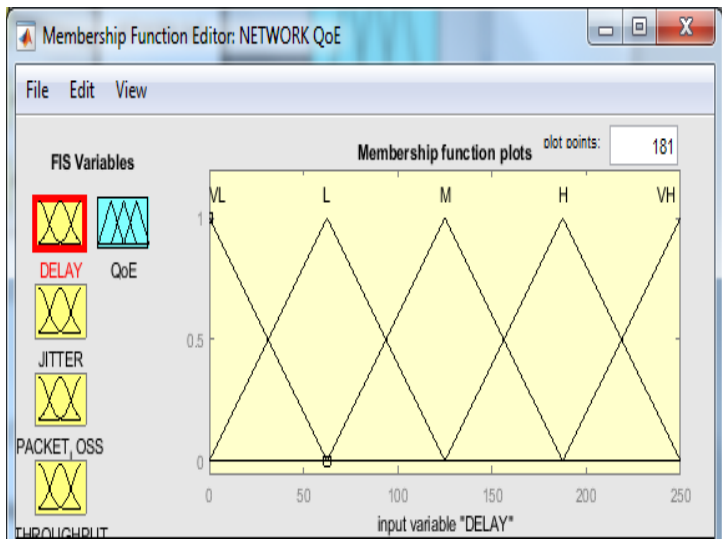

Figure 4.17: Developed membership function plots for

Delay input linguistic term.

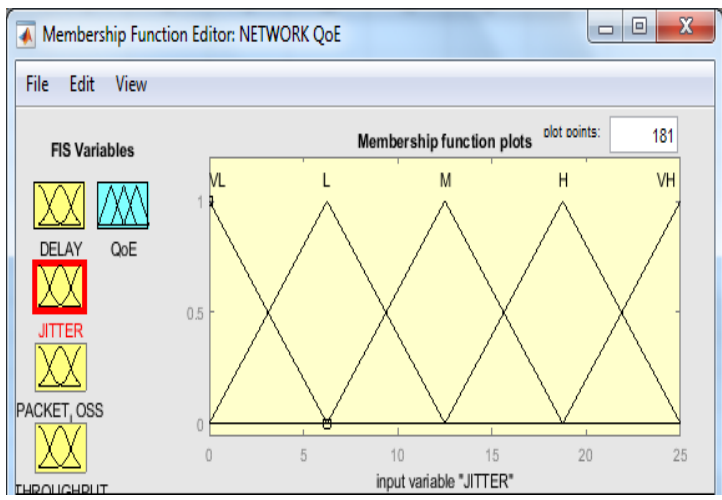

Figure 4.18: Developed membership function plots for Jitter input linguistic term. 


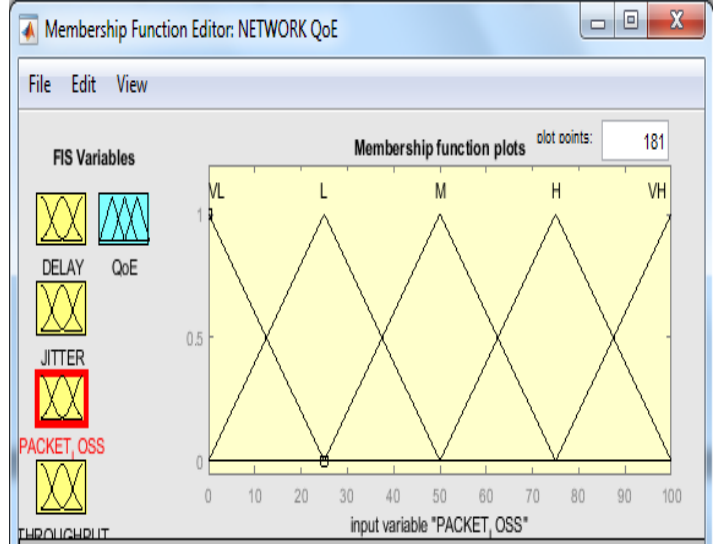

Figure 4.19: Developed membership function plots for

Packet loss input linguistic term.

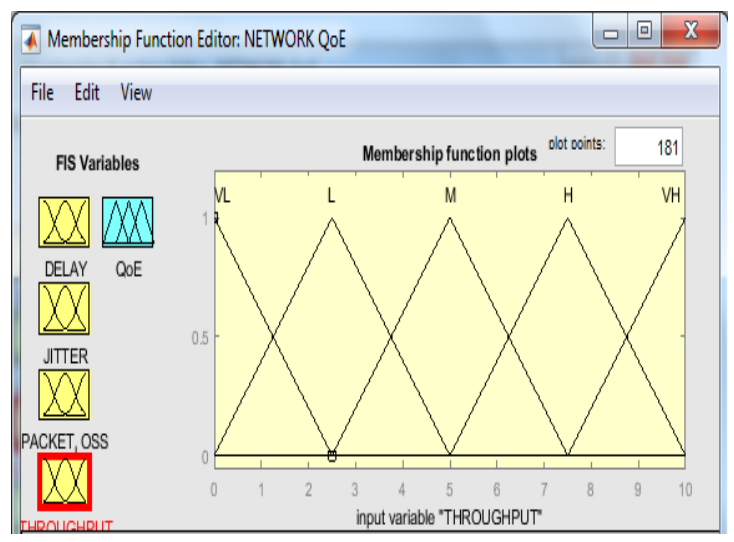

Figure 4.20: Developed membership function plots for

Throughput input linguistic term.

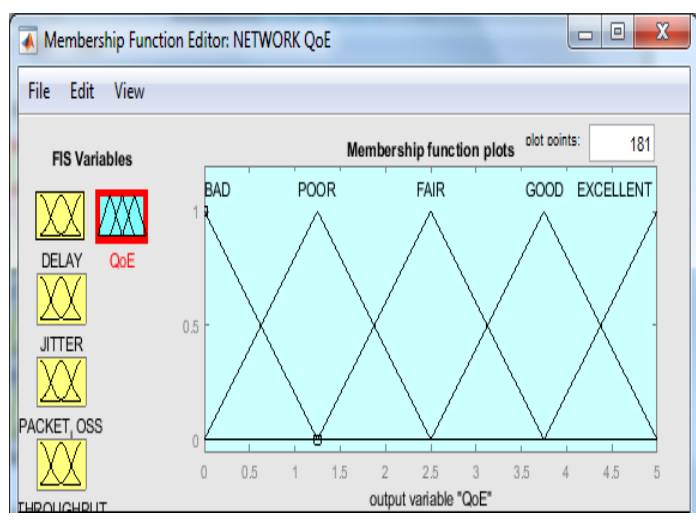

Figure 4.21: Developed membership function plots for different output linguistic terms.
7.4 To Test the Performance Of Network QoE And Estimate the Variation Of User Satisfactions Level In Function Of Network Integrity Of Service Parameters:

This objective was achieved by testing the developed framework under different input crisp values of network integrity of service parameter values while observing the performance of the framework based on the obtained QoE values.

This activity was performed in the Matlab's Rule Viewer as shown below.

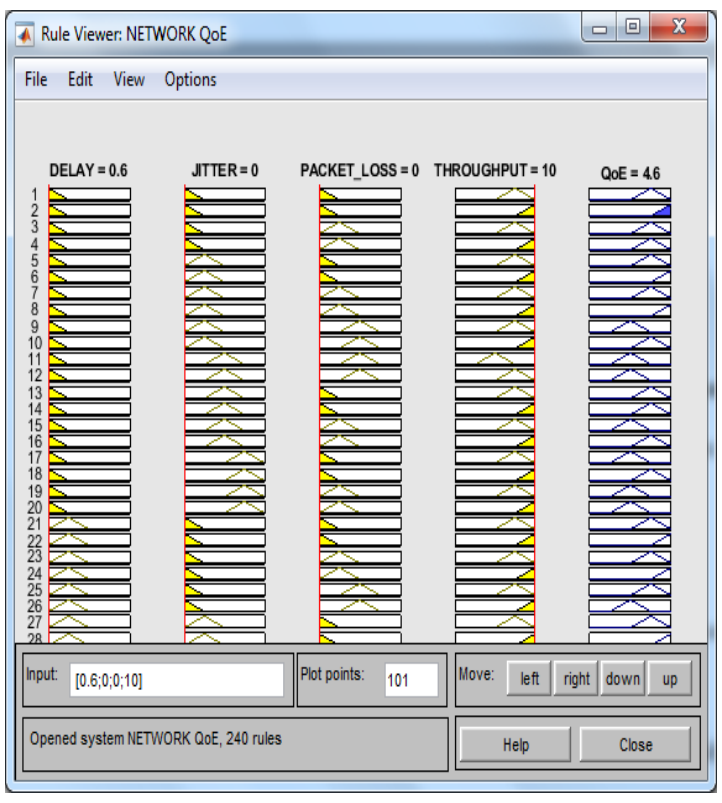

Figure 4.22: Developed Framework Testing environment in the rule viewer.

\subsection{To Implement the Developed} Framework For Use By End Users:

This activity was achieved by the help of "guide" command in matlab to develop a Graphical User Interface (GUI).Once the command is run, it prompts for an option to either create GUI or Opening an existing GUI. In this scenario, there was need to create a new GUI which when saved into the selected folder it resulted into two file formats i.e. .fig (to access the underlying 
objects in the figure) and .m file formats (To indicate

MATLAB code is in files with extension .M)

This objective was achieved by developing a framework that is executable having the capability to be installed in computing devices and perform network QoE analysis based on the inputted data as shown below:

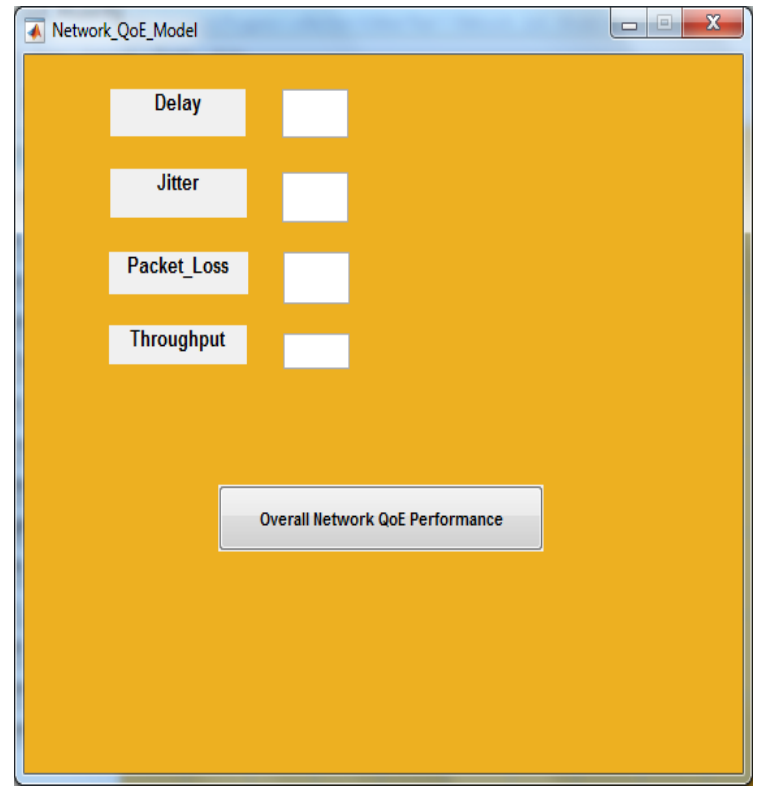

Figure 4.23: Developed User interface.

\section{CONCLUSION:}

Based on summary of work done in this research, in conclusion this research work successfully designed, developed, tested and implemented a computer networks QoE framework based on fuzzy logic methodology. The framework analyses the QoS provided by the service providers as perceived by the end users to be used for decision making in order to achieve QoS as per service level agreement. For this scenario, the model was intended for Internet Service Providers to analyze the products \& services they deliver to their clients including internet services though the framework can be customized to suite other product/service provider industries. The framework allows users to use qualitative method (Fuzzy Logic concepts) instead of quantitative method (MOS) for analysis of computer networks QoE. This allows vagueness and subjectivity nature into the developed framework.

This work intensified in the underlying QoS-related parameters, which are linked to the integrity of service QoE parameters as the area of study. These are the primary factor for QoS quantification of any network [14].

\section{RECOMMENDATIONS.}

Based on the conclusion, it is greatly recommended to adopt Frameworks that allows users to use qualitative methods for instance Fuzzy Logic concepts that have capabilities to accept vague and subjective values for analysis and decision making based on certain concepts or methodology.

Quantitative methods for instance use of Mean Opinion Score for analysis of QoE has a drawback in that user satisfaction is not precise rather it's subjective in nature hence difficult to be quantified using such methods.

\section{KNOWLEDGE CONTRIBUTION TO THE FIELD OF STUDY.}

Based on this research work, some of the accomplished tasks have contributed some knowledge in this field of study. This is clearly outlined whereby, basing on previous studies, few models exists that measure qualitative analysis of computer network quality of experience. However none incorporated all the four parameters of integrity of service; throughput, delay, packet loss and jitter as parameters of network QoE. The study's objective is to address this gap by developing a fuzzy logic model for analysis of computer network QoE basing on all the four parameters for integrity of service to check for efficiency of the model.

Moreover, the experiment phase happened to gather relevant data using Linux mtr tool. This data was the hardest part to crack through, thus hindering many research works to be completed. Data to do with networks can be best acquired from service providers. Unluckily, this data can't be rendered to the public for use or analysis due to security reasons and privacy issues. Therefore, having captured the live data through experiment, at this juncture I can avail it in public domain using various sites for instance Git-hub to assist other researchers to accomplish their work too in this field of study.

\section{FUTURE WORK/FURTHER GAPS IN RELATED RESEARCH.}

Future work in this work can be accomplished by evaluating the best Fuzzy inference system applicable to network QoE analysis for instance to compare the output results obtained by both Mamdani and sugeno fuzzy inference systems. 
Based on the conclusion, this work intensified in the underlying QoS-related parameters, which are linked to the integrity of service QoE parameters as the area of study. These are the primary factors for QoS quantification of any network [14]. Since the Accesibility and retainability QoE parameters have not been tackled, future work can incorporate their respective underlying QoS-related parameters into the model to test for efficiency of the model.The respective underlying parameters are represented below;

TABLE 2.1: Mapping between QoE and QoS Related parameters [15]

\begin{tabular}{|l|l|}
\hline QoE parameters & \multicolumn{1}{|c|}{$\begin{array}{c}\text { Underlying QoS-related } \\
\text { parameters }\end{array}$} \\
\hline Accesibility & $\begin{array}{l}\text { Unavailability } \\
\text { Security } \\
\text { Activation } \\
\text { Access } \\
\text { Coverage } \\
\text { Blocking } \\
\text { Setup time }\end{array}$ \\
\hline Retainability & Connection loss \\
\hline Integrity of Service & $\begin{array}{l}\text { Throughput } \\
\text { Delay } \\
\text { Delay variation/Jitter } \\
\text { Packet loss }\end{array}$ \\
\hline
\end{tabular}

\section{ACKNOWLEDGEMENT}

Firstly, I would like to acknowledge God, the creator of heaven and earth for the gift of wisdom to think towards this research work. I would like to express my deep gratitude to my thesis supervisors; Dr. Calvin Otieno $(\mathrm{PhD})$, for his patient guidance, enthusiastic encouragement and keeping my progress on schedule. Dr. Richard Rimiru ( $\mathrm{PhD}$ ), for his advice, assistance in keeping my progress on schedule and useful critiques of this research work.

My grateful thanks are also extended to Mr.George Ochieng, the Chair of IT department at JKUAT Mombasa CBD for ensuring we are exposed to several presentation seminars as one of the requirements before being awarded with master's degree, to Mr.Abdulrehman Ahmed who helped me in documenting my work and provision of technical advice and to Mrs. Amina Omar for her support in Matlab environment. My sincere appreciation also goes to all my lecturers who took me through this journey of academics, support staff including the Librarian for provision of access to research materials.

I would also like to extend my thanks to Technical University of Mombasa (TUM) laboratory technician, Mr.Aggrey Shitsukane for his inclusive support for provision of access to Laboratory environment at TUM in order to carry out my research work with precision.

Finally, I wish to thank my Family and Friends for their support and encouragement throughout my study. May God bless you all and guide you with these wise Quotes;

Start where you are. Use what you have. Do what you can. Arthur Ashe. Moreover, there are no traffic jams on the extra mile. - Zig Ziglar.

\section{REFERENCES}

[1] K. Brunnstr et al., "Qualinet White Paper on Definitions of Quality of Experience Qualinet White Paper on Definitions of Quality of Experience Output from the fifth Qualinet meeting, Novi Sad ," 2014.

[2] ITU, "P.800: Methods for subjective determination of transmission quality," ITU-T Recomm., vol. 800, 1996.

[3] L. A. Zadeh, I. Introduction, and U. S. Navy, "Fuzzy Sets *-," vol. 353, pp. 338-353, 1965.

[4] A. S. Omar, M. Waweru, and R. Rimiru, "Application of Fuzzy Logic in Qualitative Performance Measurement of Supply Chain Management," vol. 5, no. 6, 2015.

[5] M. Madhoushi and A. N. Aliabadi, "Environmental Performance Evaluation Based on Fuzzy Logic," Int. J. Appl. Sci. Technol., vol. 1, no. 5, pp. 432-436, 2011.

[6] E. Olugu, "Supply Chain Performance Evaluation: Trends and Challenges Supply Chain Performance Evaluation: Trends and Challenges," no. January 2009,2016

[7] R. Hawi, G. Okeyo, and M. Kimwele, "Techniques for Smart Traffic Control : An In-depth Review," vol. 4, no. 7, pp. 566-573, 2015.

[8] A. Hamam, M. Eid, A. El Saddik, and N. D Georganas, "A Fuzzy Logic System for Evaluating Quality of Experience of Haptic-Based Applications," no. October 2016, 2008.

[9] J. Pokhrel, "Intelligent quality of experience ( QoE ) 
International Journal of Computer Applications Technology and Research

Volume 8-Issue 04, 135-148, 2019, ISSN:-2319-8656

analysis of network served multimedia and web contents Analyse intelligente de la qualité d , expérience ( QoE ) dans les réseaux de diffusion de contenu Web et Multimédia,” 2015.

[10] S. M. Ataeian and M. J. Darbandi, "Analysis of Quality of Experience by applying Fuzzy logic A study on response time," no. June, 2011.

[11] O. F. W. Onifade, "Better Quality of Service Management With Fuzzy Logic In Mobile Adhoc Network," vol. 6, no. 1, pp. 59-68, 2013.

[12] M. E. A. Ebrahim and H. A. Hefny, "Fuzzy Logic based Approach for VoIP Quality Maintaining," vol. 9, no. 1, pp. 537-542, 2018.

[13] P. Singhala, D. N. Shah, and B. Patel, "Temperature Control using Fuzzy Logic,” vol. 4, no. 1, pp. 1-10, 2014.

[14] Y. Chen, T. Farley, and N. Ye, "QoS Requirements of Network Applications on the Internet," vol. 4, pp. 55-76, 2004.

[15] F. Farid, S. Shahrestani, and C. Ruan, "A Fuzzy Logic Approach for Quality of Service Quantification in Wireless and Mobile Networks," pp. 629-636, 2014. 